\title{
Small Signal Interactions Involving a Synchronous Machine and a Grid Forming Converter
}

\author{
Luke Benedetti, Panagiotis N. Papadopoulos, Agustí Egea-Alvarez \\ Department of Electronic and Electrical Engineering \\ University of Strathclyde \\ Glasgow, UK \\ Luke.Benedetti@strath.ac.uk
}

\begin{abstract}
Interactions between synchronous machines (SMs) and grid forming converters (GFMs) will become increasingly relevant as the power system transitions from a conventional fossil fueled synchronous based system to a renewable generation rich and converter-based system. This paper investigates and confirms the possibility of electromechanical oscillatory modes between conventional SMs and converter connected generation using a GFM control algorithm as well as between two GFMs. The study employs small-signal models and performs small-signal eigenvalue analysis. Moreover, parametric sweeps are performed, to investigate the impact of network and GFM control parameters in controlling the electromechanical modes.
\end{abstract}

Index Terms-Control, dynamics, electromechanical oscillations, grid-forming converter, power electronics, power systems, small signal stability, virtual synchronous machine.

\section{INTRODUCTION}

The urgency associated with climate action is well understood, and the acceleration of the integration of renewable energy sources (RESs) within the electricity grid is becoming a necessity if global temperature levels are to remain within acceptable levels [1]. Power converters are required to interface most RESs to the electricity system and due to their very different characteristics, can potentially introduce stability issues related to the reduction of inertia, voltage and frequency regulation, and loss of synchronizing torque [2], amongst other challenges. GFMs are one of the recommended approaches to facilitate the transition from conventional synchronous generation to converter interfaced generation (CIG).

GFM is a term incorporating a grid-tied converter controlled with the specific inclusion of voltage and frequency regulation [3]. This is different from the grid following converters (GFLs) which track the voltage signal and inject the specified power. There is a broad range of control philosophies under the GFM heading, each with several different implementations and benefits. Some of the most popular schemes currently within the literature include droop control [4], virtual oscillator control (VoC) [5] and the classification most similar to the generic GFM used in this investigation, the VSM [6].

There are several references in the literature, investigating the impact of a high penetration of CIG on power systems [7], but these are typically looking at gridfollowing converters as opposed to GFMs. In addition, there are several references describing different GFM approaches

978-1-6654-3597-0/21/\$31.00 C2021 IEEE and implementations. However, there is limited research into the impact of these technologies on the stability of the power system as a whole.

Small-signal analysis of GFMs in parallel connected to an infinite bus has been investigated in [8]. Additional small signal analysis looking at robust stability margins using the $\mu$ factor has been performed by the same authors in [9]. These papers look at synchronverters, a type of swing equation based VSM, operating in parallel as well as synchronverters in parallel with GFLs.

A simplified frequency analysis for $100 \%$ CIG systems has been outlined in [10]. The approach exploits similarities between the SM and GFMs by allowing for the aggregation of multiple machines. This is possible by considering the similarities between the swing equation and the GFM control structure, which is different to the one used in this study. However, they do not provide a small-signal analysis of the electromechanical oscillations themselves.

A detailed small-signal analysis has been performed in [11]. In this study the authors use eigenvalue analysis to determine when a system comprised of two machines (all combinations of SMs, GFLs and GFMs) become unstable with increasing penetrations of the CIG based machine (and of the GFL specifically in the GFM-GFL case). It is seen from participation factor analysis that there are destabilising interactions between the fast voltage control of the converters and the relatively slow voltage control of the PSS and AVR of the SM. It is also found that as GFL penetration increases there reaches a point where there is not enough contribution from the SM (or GFM) to slow the frequency variations and therefore the phase-locked loop (PLL) of the GFL loses synchronism. They also explore the impact of transmission line dynamics and the power system operating point and expand their study to larger systems: first the IEEE-9 bus and then a modified version of the South-East Australian network. However, again, electromechanical modes involving GFMs are not discussed in detail.

From the literature, it can be determined that with the inclusion of GFMs, further consideration to dynamic interactions needs to be given, since new instability mechanisms might appear in a complex power system. Therefore, considering power systems will most probably involve SMs for years to come, the interactions between

The work of Luke Benedetti was supported by an EPSRC Student Excellence Award Studentship. The work of Panagiotis Papadopoulos was supported by a UKRI Future Leaders Fellowship (grant number: MR/S034420/1). All results can be fully reproduced using the methods and data described in this paper and provided references. 
CIG and SMs should be well understood. Additionally, with GFMs being recognised as a potential solution for maintaining stability, interactions specifically between SMs and GFMs should be studied carefully.

The fact that GFMs control active power through the voltage angle, and have a resultant inherent frequency synchronization, suggests the possibility of electromechanical interactions between multiple machines [10]. Therefore, in this paper an initial investigation into interactions between a GFM and an SM as well as between two GFMs is performed. To the extent of our knowledge, this particular interaction has not been investigated, excluding qualitative recognition that this phenomenon is to be expected. Moreover, the impact of GFM control gains and transmission line lengths on such oscillatory modes and interactions between SMs and GFMs is also investigated in this paper. It should be noted that the EMT implementation of the models in this work allow for easier adaptation for future investigations into more complicated or detailed systems and components, despite this level of detail not being necessary to identify electromechanical modes of oscillation. This future adaptability was considered because high frequency phenomena have been proven to be significant in the stability of systems with substantial levels of CIG [8], [11], [12]. This paper is mainly interested in investigating electromechanical modes, hence a relatively simple (low order) SM model is used. However, a series of high frequency oscillatory modes closely associated with transmission line dynamics are also identified, one of which is found to be of interest in terms of stability limits. This investigation, and the SM model used within, is considered a preliminary step applied on a smaller system to more easily identify and investigate such interactions. A modular modelling approach is adopted to allow for easy expansion to lager systems and higher order SM model as part of future work.

The remainder of this paper is structured as follows: section II describes the modelling of the network components and details the layout of the system; section III discusses the results and analysis of the performed simulations and section IV provides the conclusions.

\section{Methodology AND Modelling}

The small signal modelling in this work involves identifying the nonlinear state-space models of each component and considering how they connect together to form a wider network. The differential algebraic equations (DAEs) associated with each component are then linearised and connected to form a single full network small signal model (SSM). This section briefly describes the linearisation process and then proceeds to detail the models of each component used within this study.

\section{A. Linearisation Process}

A common approach to study electromechanical (and any other relevant) modes associated with a machine or interactions between multiple machines is to perform a small-signal analysis. To achieve this, the system being analysed must be linear, but power systems are known to be highly nonlinear and complex. Therefore, it must first be linearised around the steady state operating point. First, the DAEs of the system are arranged in state-space form as,

$$
\begin{aligned}
& \dot{\mathbf{x}}=\mathbf{A x}+\mathbf{B u}=\mathbf{f}(\mathbf{x}, \mathbf{u}) \\
& \mathbf{y}=\mathbf{C x}+\mathbf{D u}=\mathbf{g}(\mathbf{x}, \mathbf{u})
\end{aligned}
$$

where $\boldsymbol{x}$ is the vector of state variables and $\boldsymbol{u}$ and $\boldsymbol{y}$ the vectors for the inputs and outputs, respectively. The dot above the state vector in (1) suggests differentiation with respect to time. $\boldsymbol{A}$ is the state matrix, $\boldsymbol{B}$ is the control matrix, $\boldsymbol{C}$ is the output matrix and $\boldsymbol{D}$ is the feedforward matrix.

The linearisation is then achieved by introducing a small disturbance, denoted by the prefix $\Delta$, performing the Taylor series expansion and then discarding all terms above first order, resulting in small deviation equations of the form,

$$
\begin{gathered}
\Delta \mathrm{x}_{\mathrm{i}}=\frac{\partial \mathrm{f}_{\mathrm{i}}}{\partial \mathrm{x}_{1}} \Delta \mathrm{x}_{1}+\ldots+\frac{\partial \mathrm{f}_{\mathrm{i}}}{\partial \mathrm{x}_{\mathrm{n}}} \Delta \mathrm{x}_{\mathrm{n}}+\frac{\partial \mathrm{f}_{\mathrm{i}}}{\partial \mathrm{u}_{1}} \Delta \mathrm{u}_{1}+\ldots+\frac{\partial \mathrm{f}_{\mathrm{i}}}{\partial \mathrm{u}_{\mathrm{r}}} \Delta \mathrm{u}_{\mathrm{r}} \\
\Delta \mathrm{y}_{\mathrm{k}}=\frac{\partial \mathrm{g}_{\mathrm{k}}}{\partial \mathrm{x}_{1}} \Delta \mathrm{x}_{1}+\ldots+\frac{\partial \mathrm{g}_{\mathrm{k}}}{\partial \mathrm{x}_{\mathrm{n}}} \Delta \mathrm{x}_{\mathrm{n}}+\frac{\partial \mathrm{g}_{\mathrm{k}}}{\partial \mathrm{u}_{1}} \Delta \mathrm{u}_{1}+\ldots+\frac{\partial \mathrm{g}_{\mathrm{k}}}{\partial \mathrm{u}_{\mathrm{r}}} \Delta \mathrm{u}_{\mathrm{r}}
\end{gathered}
$$

It should be noted that intermediate steps for understanding have been omitted but the full procedure can be found in [13].

\section{B. Component Modelling}

The component models included in this investigation are a SM, a GFM, RL branch transmission lines and a static constant impedance RL load.

\section{1) Modelling Convention}

The state-space equations are derived in the dq0 frame with each machine operating in its own reference frame dependent on its rotor angle, or virtual rotor angle for the GFM. The common reference frame can be chosen in one of two ways. The first is to determine the 'centre of inertia' and the second is to simply chose a machine to be the reference. The second approach was adopted for this investigation because it offers easier implementation with no requirement to parameterise the inertia constant and damping of the GFM. Additionally, the second approach avoids the resultant redundant eigenvalue of the first approach [13].

Since the whole model is implemented in dq0 terms, whenever a Park transform would have been required to transform signals in the abc-frame to the dq0-frame, there is instead a simple vector rotation dependent on the angle between the two dq0 frames. The same is true for any instance of an inverse Park transform.

Several different modules within the network contain series RL or parallel capacitive components. In the balanced $\mathrm{dq} 0$ frame, these can be realised with equations:

$$
\dot{\mathrm{i}_{\mathrm{dq}}}=\frac{1}{\mathrm{~L}}\left(\mathrm{v}_{1_{\mathrm{dq}}}-\mathrm{v}_{\mathrm{2}_{\mathrm{dq}}}\right)-\left(\frac{\mathrm{R}}{\mathrm{L}}+\mathrm{j} \omega\right) \mathrm{i}_{\mathrm{dq}}
$$




$$
\mathrm{v}_{\mathrm{dq}}=\frac{1}{\mathrm{C}}\left(\mathrm{i}_{\mathrm{dq}}-\mathrm{i}_{2_{\mathrm{dq}}}\right)-\mathrm{j} \omega \mathrm{v}_{\mathrm{dq}}
$$

where the currents, voltages, resistance, and inductance are displayed in figure 1. Note, the subscript ' $d q$ ' denotes the phasor represented by $\mathrm{d}$ and q components as $x_{d q}=x_{d}-$ $j x_{q}$. The equations for the RL and the $\mathrm{C}$ segments can be extracted and used separately depending on the configuration. For example, the transmission lines are implemented as simple RL impedances modelled in accordance with (5) but neglecting the capacitive dynamics described by (6).

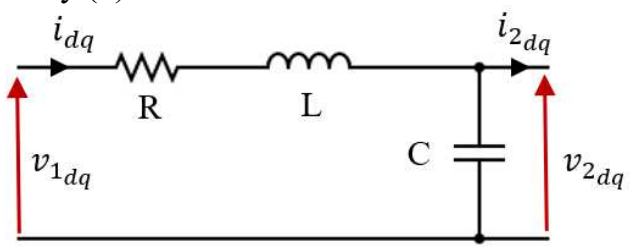

Figure 1. RLC circuit diagram.

\section{2) Synchronous Machine}

The SM model comprises the swing equation along with an RL impedance but neglects rotor dynamics as in this paper we are focusing on identifying oscillatory modes in the electromechanical range. The swing equation captures the relationship between the SM rotor speed (and hence angle) and the difference between the input mechanical and output electrical power. This relationship can be written as,

$$
\begin{gathered}
\dot{\omega}_{\mathrm{r}}=\frac{1}{2 \mathrm{H}}\left(\mathrm{P}_{\mathrm{m}}-\mathrm{P}_{\mathrm{e}}-\mathrm{K}_{\mathrm{D}} \Delta \omega-\mathrm{K}_{\mathrm{gov}} \Delta \omega\right) \\
\dot{\delta}_{\mathrm{r}}=\omega_{\mathrm{r}}
\end{gathered}
$$

where $\omega_{r}$ and $\delta_{r}$ are the rotor speed and angle, respectively. The damping coefficient is termed $K_{D}$ and the inertia constant, $H$. Finally, the mechanical and electrical powers are denoted by $P_{m}$ and $P_{e}$, respectively. A speed governor has been included which has been simplified to a proportional gain, $K_{g o v}$, acting on a change of rotor speed to augment the mechanical input power.

The electrical part of the SM model consists of the resistance and inductance resulting from the armature coils in addition to the inductance attributed to the armature reaction. The combined impedance is termed the synchronous impedance, $Z_{s}=R_{s}+j X_{s}$, with $R_{s}$ being the armature resistance and $X_{S}$ combining the effects of the armature leakage inductance and armature reaction. The transformation from the $\mathrm{SM} \mathrm{dq0}$-frame to the common dq0frame is performed behind the synchronous impedance. However, there is a possible alternative whereby the transformation is performed at the SM terminals (after the synchronous impedance). This synchronous impedance is modelled dynamically, in accordance with (5).

\section{3) Grid Forming Converter}

The power converter section of the GFM is represented with an averaged model which neglects switching effects and the time delay usually associated with the employment of pulse width modulation (PWM). An inverse Park transform is used to translate the converter voltage and angle from the control scheme directly into the voltage at the output of the converter. There is also a harmonic filter containing a series RL impedance followed by a parallel capacitance at the GFM output terminal. This is of the configuration seen in figure 1 . and modelled in accordance with (5) and (6).

The control structure of the GFM contains two main loops. The first is the power loop which manipulates the virtual rotor speed, and hence angle, with a PI controller acting on the difference between the reference power $P_{\text {ref }}$ and the measured (or feedback) power $P_{f b}$. This is the same principle as the SM swing equation and the two systems can be directly compared in their second order dynamic responses by looking at the characteristic equation of their transfer functions. This allows for the equivalent inertia and damping values to be described in terms of the PI controller gains, $K_{I}$ and $K_{P}$. From this it is found that $K_{I}$ impacts inertia while both $K_{I}$ and $K_{P}$ impact damping. However, it can be noted that there are differences in the steady state response since the PI controller acts to bring the output power exactly to its reference, that is there is no damping feedback term acting on the change of rotor speed as there is in the swing equation (although the red droop branch in fig. 2 essentially solves this). The second loop is the voltage loop, acting as an AVR by maintaining the voltage magnitude at the filtering capacitor, $V_{f b}$, to the reference value, $V_{r e f}$.

In the test case with only GFMs, described later, one of the machines is also equipped with frequency droop control to balance the active power in the system. The block diagram for the GFM control structure is displayed in fig. 2. Note, the frequency droop branch (displayed in red) is only present for one of the machines in the only-GFM test case, for better equivalence to the SM-GFM test case.

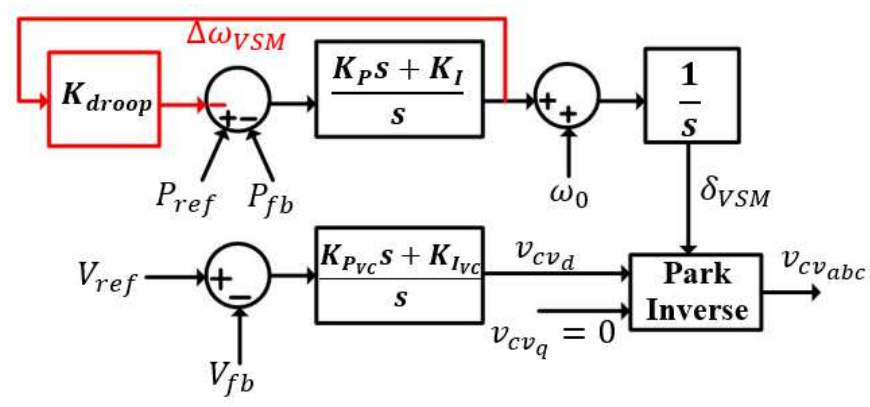

Figure 2. GFM control scheme block diagram.

4) Static Load

The load is modelled as a constant RL impedance. The values of the resistance and inductance are calculated with

$$
\begin{gathered}
\mathrm{R}_{\text {load }}=\mathrm{V}_{\mathrm{n}}^{2} / \mathrm{P}_{\text {load }} \\
\mathrm{L}_{\text {load }}=\mathrm{V}_{\mathrm{n}}^{2} /\left(\omega_{0} \times \mathrm{Q}_{\text {load }}\right)
\end{gathered}
$$


where $V_{n}$ is the base voltage and $\omega_{0}$ is the base electrical frequency. The desired load active and reactive powers are denoted by $P_{\text {load }}$ and $Q_{\text {load }}$, respectively.

\section{Systems Under Study}

Two networks with the layout in fig. 3 are analysed in this work. The 'swing' machine being the SM in one test case and the droop-augmented GFM in the other. Further signals within the machines include $i_{c v_{d q}}$ which is the current through the RL section of the GFM output filter and $i_{s m_{d q}}$ which is simply equivalent to $i_{t l l d q}$ in this case. Additionally, $v_{c v_{d q}}$ is the voltage behind the filter impedance in the GFM and $E_{d q}$ is the internal generated voltage of the SM. In the GFM-GFM network, all GFM specific parameters or signals are given a subscript of ' 1 ' if related to the left machine or ' 2 ' if related to the right. The modularity of the networks is achieved through development of state space models for each component separately (including individual transmission line branches). Following this, the interconnection of signals is achieved with an additional module pertaining to voltage specification and Kirchhoff's current law for each bus. Finally, the initial states required for the SSMs were obtained using a power flow analysis with the aid of MATPOWER to solve the steady-state equations.

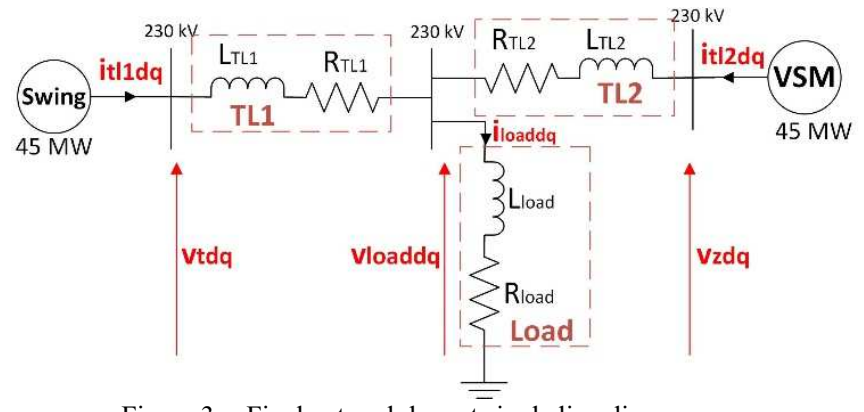

Figure 3. Final network layout single line diagram.

The SSMs have been validated by comparing step responses with corresponding Simulink models.

\section{Small Signal Analysis}

For both network configurations being tested, small-signal analysis utilising eigenvalues is performed. The use of eigenvalue analysis [13] offers insight into the oscillatory modes that might be excited after a disturbance such as a load increase. With this, potential electromechanical modes can be identified by calculating the frequency of the modes and extracting those in the proximity of up to $3 \mathrm{~Hz}$. Typically, with SMs, local modes are in the range of 1 to 3 $\mathrm{Hz}$ and interarea modes are less than $1 \mathrm{~Hz}$ [14]. The eigenvalues and associated frequency and damping ratio are obtained as in [13]. The next step is to calculate the participation factor of each state for each mode. This gives an idea of which states are the most involved in specific oscillatory modes and is especially useful in identifying interactions between two machines. The calculation, from [14], is

$$
\mathrm{p}_{\mathrm{ij}}=\left(\left|\psi_{\mathrm{ij}}\right| \times\left|\phi_{\mathrm{ij}}\right|\right) /\left(\sum_{\mathrm{i}=1}^{\mathrm{n}}\left|\psi_{\mathrm{ij}}\right| \times\left|\phi_{\mathrm{ij}}\right|\right)
$$

where $\psi$ and $\phi$ are the left and right eigenvectors, respectively.

Finally, parametric sweeps are performed to determine the impact on the small-signal stability of different network elements such as transmission line lengths or GFM controls. The parameters associated with each test case are displayed in table i. The GFM parameters are common to both machines in the only-GFM network with the swing machine also having a droop parameter of $K_{\text {droop }}=0.5\left(100 \times 10^{6}\right)$.

TABLE I. NETWORK PARAMETERS.

\begin{tabular}{|c|c|c|c|}
\hline SM Parameter & Value & $\begin{array}{c}\text { GFM } \\
\text { Parameter }\end{array}$ & Value \\
\hline$H$ & 4 & $K_{P}$ & $9 \times 10^{-9}$ \\
\hline$K_{D}+K_{\text {gov }}$ & $\cong 10$ & $K_{I}$ & $4 \times 10^{-8}$ \\
\hline$R_{S}$ & $10.58 \Omega$ & $K_{P_{V C}}$ & 0.1 \\
\hline$L_{S}$ & $0.5052 \mathrm{H}$ & $K_{I_{V C}}$ & 50 \\
\hline$P_{m}$ & $50 \mathrm{MW}$ & $R_{f}$ & $5.29 \Omega$ \\
\hline$V_{\text {ref }}$ & $230 \mathrm{kV}$ & $L_{f}$ & $0.1347 \mathrm{H}$ \\
\hline $\begin{array}{c}\text { Network } \\
\text { Parameter }\end{array}$ & Value & $C_{f}$ & $1 \mu \mathrm{F}$ \\
\hline$R_{T L I}=R_{T L 2}$ & $35.688 \Omega$ & $P_{r e f}$ & $50 \mathrm{MW}$ \\
\hline$L_{T L I}=L_{T L 2}$ & $0.1272 \mathrm{H}$ & $V_{\text {ref }}$ & $230 \mathrm{kV}$ \\
\hline
\end{tabular}

\section{RESULTS AND ANALYSIS}

\section{A. SM-GFM Network Eigenvalue Analysis}

The procedure explained above is performed for the network containing the SM and GFM combination. The eigenvalues are presented in table ii. This table also includes the frequency of the mode and the corresponding damping ratio. Using this information, the electromechanical mode is identified as $\lambda_{9} \& \lambda_{10}$. Following this, the participation factors representing the contribution of each state to each oscillatory mode were calculated and those with significant contribution $(>10 \%)$ were added to the table.

TABLE II. SM-GFM-LOAD SYSTEM EIGENVALUES.

\begin{tabular}{|c|c|c|c|c|}
\hline Eigenvalues & Value & Frequency & $\begin{array}{c}\text { Damping } \\
\text { Ratio }\end{array}$ & $\begin{array}{c}\text { Contributing } \\
\text { States }\end{array}$ \\
\hline$\lambda_{1} \& \lambda_{2}$ & $\begin{array}{c}-9.56 \cdot 10^{3} \\
\pm j 8.42 \cdot 10^{4}\end{array}$ & $13.396 \mathrm{kHz}$ & $11.28 \%$ & $i_{s m_{d q}}, i_{t l_{d q}}$ \\
\hline$\lambda_{3} \& \lambda_{4}$ & $-77.07 \pm j 3843$ & $612 \mathrm{~Hz}$ & $2.01 \%$ & $v_{z_{d q}}, i_{c_{d q}}$ \\
\hline$\lambda_{5} \& \lambda_{6}$ & $-52.38 \pm j 3043$ & $484 \mathrm{~Hz}$ & $1.72 \%$ & $v_{z_{d q}}, i_{c v_{d q}}$ \\
\hline$\lambda_{7} \& \lambda_{8}$ & $\begin{array}{l}-216.15 \\
\pm j 72.71\end{array}$ & $115 \mathrm{~Hz}$ & $28.65 \%$ & $\begin{array}{c}i_{c v_{d q}}, i_{s m_{d q}}, \\
i_{t l l_{d q}}\end{array}$ \\
\hline$\lambda_{9} \& \lambda_{10}$ & $\begin{array}{c}-1.5661 \\
\pm j 10.57\end{array}$ & $1.68 \mathrm{~Hz}$ & $14.65 \%$ & $\omega_{r}, \delta_{G F M}$ \\
\hline
\end{tabular}

The most significant states in contributing to the electromechanical mode are $\omega_{r}$ and $\delta_{G F M}$. This clearly suggests an interaction between the SM and GFM, 
confirming the expected behaviour. This information is useful in considering future control of power systems.

Techniques previously used to address interactions between SMs will likely need to be considered as GFM-coupled RESs are integrated.

High frequency oscillatory modes are also present, the most interesting being $\lambda_{3}$ to $\lambda_{6}$ which are seen to have very low damping. Despite the low damping ratio associated with some of these modes, they are damped very quickly in time. The damping ratio represents attenuation of the mode per cycle and with high frequency, the oscillation does not last long in time. Through parametric sweeps (excess to those in the scope of this paper), the eigenvalues of $\lambda_{3}$ to $\lambda_{6}$ were affected by the GFM voltage loop controls as well as the transmission line lengths, as expected from the contributing states which includes a small participation from the voltage loop integrator state, $V_{\text {int }}$, of $0.19 \%$ for $\lambda_{3}$ $\& \lambda_{4}$ and $0.27 \%$ for $\lambda_{5} \& \lambda_{6}$.

The very fast oscillatory mode of $13.396 \mathrm{kHz}$ was only found to be affected by the transmission line length and not by any of the GFM control gains. Also, the remaining mode of $115 \mathrm{~Hz}$ is discussed later with the $K_{P}$ parametric sweep.

\section{B. GFM-GFM Network Eigenvalue Analysis}

In a similar manner, the eigenvalues and corresponding attributes for the GFM-GFM network are presented in table III. The electromechanical mode is this time identified to be $\lambda_{13} \& \lambda_{14}$. Again, the participation factors are calculated and contributing states of each mode are shown in table iii. The states with the highest contribution to the electromechanical modes are found to be $P_{\text {int }}, P_{\text {int }}$, and $\delta_{V S M_{2}}$. These states are associated with the active power loops of the GFMs and suggest an electromechanical interaction. High frequency modes are also present in this network with analysis being
TABLE III. GFM-GFM-LOAD SYSTEM EIGENVALUES.

\begin{tabular}{|c|c|c|c|c|}
\hline Eigenvalues & Value & Frequency & $\begin{array}{c}\text { Damping } \\
\text { Ratio }\end{array}$ & $\begin{array}{c}\text { Contributing } \\
\text { States }\end{array}$ \\
\hline$\lambda_{1} \& \lambda_{2}$ & $\begin{array}{c}-9.51 \cdot 10^{3} \\
\pm j 8.36 \cdot 10^{4}\end{array}$ & $\begin{array}{c}13.303 \\
\mathrm{kHz}\end{array}$ & $11.31 \%$ & $i_{t l_{d q}}, i_{t l 2_{d q}}$ \\
\hline$\lambda_{3} \& \lambda_{4}$ & $-73.7 \pm j 4271$ & $680 \mathrm{~Hz}$ & $1.73 \%$ & $v_{z 1_{d q}}, v_{z 2_{d q}}$ \\
\hline$\lambda_{5} \& \lambda_{6}$ & $-75 \pm j 3636$ & $579 \mathrm{~Hz}$ & $2.06 \%$ & $v_{z 1_{d q}}, v_{z 2_{d q}}$ \\
\hline$\lambda_{7} \& \lambda_{8}$ & $-12.46 \pm j 3065$ & $488 \mathrm{~Hz}$ & $0.41 \%$ & $\begin{array}{c}v_{z l_{d}}, i_{c v 1_{d}} \\
v_{z 2_{d}}, i_{c v 2_{d}}\end{array}$ \\
\hline$\lambda_{9} \& \lambda_{10}$ & $-14.52 \pm j 2514$ & $400 \mathrm{~Hz}$ & $0.58 \%$ & $\begin{array}{c}v_{z l_{q}}, i_{c v I_{q}} \\
v_{z 2_{q}}, i_{c v 2_{q}}\end{array}$ \\
\hline$\lambda_{11} \& \lambda_{12}$ & $-155.1 \pm j 316.5$ & $50.4 \mathrm{~Hz}$ & $44 \%$ & $i_{t l l_{d q}}, i_{t l 2_{d q}}$ \\
\hline$\lambda_{13} \& \lambda_{14}$ & $-2.8 \pm j 3.7$ & $0.589 \mathrm{~Hz}$ & $60.4 \%$ & $\begin{array}{c}P_{i n t t_{1}}, \\
P_{i n t_{2}}, \delta_{V S M_{2}}\end{array}$ \\
\hline
\end{tabular}

equivalent to those in the SM-GFM network but with the addition of two oscillatory modes denoted in this network by $\lambda_{7}$ to $\lambda_{10}$. These are found to have similar characteristics to $\lambda_{3}$ to $\lambda_{6}$ for both networks.

\section{Parametric Sweeps}

To further the contribution of this work, parametric sweeps were performed for several different network and control parameters to determine their impact on the oscillatory modes.

The eigenvalues of interest are displayed with non-essential modes being omitted. The first investigation increased the lengths of the transmission lines. The resistance and reactance per kilometer of both lines are chosen by calculating the required rated current and selecting from the relevant table of cable sizes [15]. Fig. 4a displays the result of varying the TL1 and TL2 lengths from 40 to $120 \mathrm{~km}$ simultaneously. The next two investigations are performed

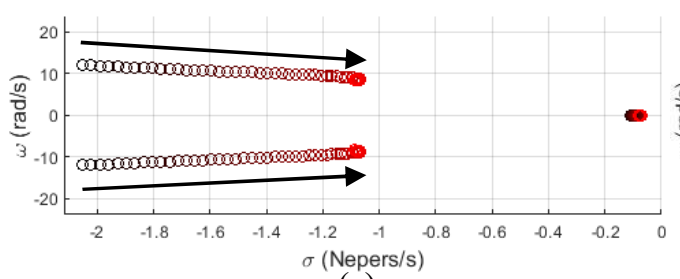

(a)

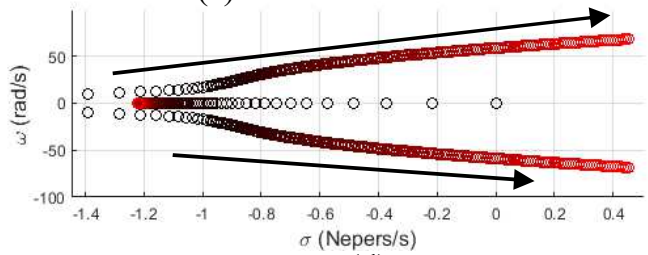

(d)

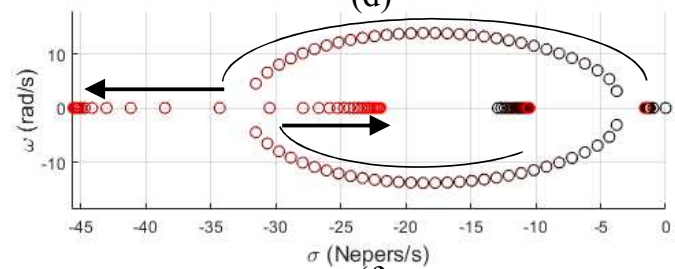

(f)

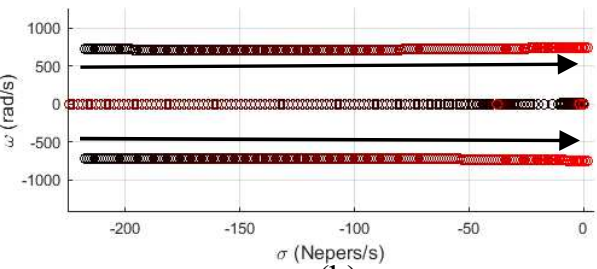

(b)

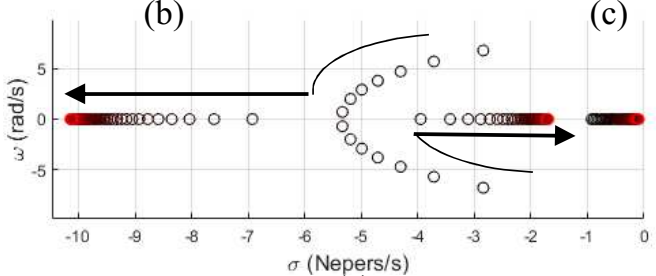

(e)

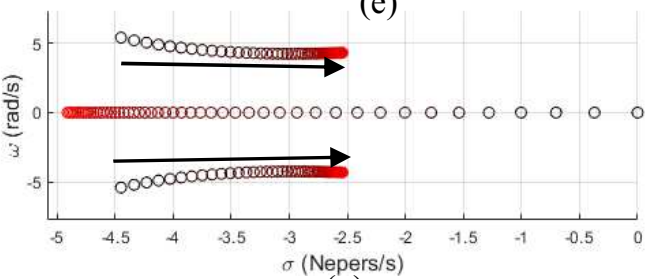

(g)

Figure 4. Eigenvalues of interest for parametric sweep of the SM-GFM-load network for (a) $T L 1 \& T L 2$, (b) $K_{P}$, (c) zoomed $K_{P}$, (d) $K_{I}$ and of the GFM-GFM-load network for (e) $K_{P I}$, (f) $K_{I I}$ and (g) $K_{\text {droop }}$. 
for the GFM power loop PI controller gains. The plots for the proportional gain, $K_{P}$, and integral gain, $K_{I}$, are displayed in fig. $4 \mathrm{~b}$ to $4 \mathrm{c}$ and fig. $4 \mathrm{~d}$, respectively. $K_{P}$ was swept from 0 to $1 \times 10^{-6}$ and $K_{I}$ was swept from $1 \times 10^{-12}$ to $1 \times 10^{-5}$. The same sweeps are performed in the GFM-GFM case and similar trends are observed; therefore, these have not been presented here. Additionally, the PI gains of the power loop for the droop-augmented-GFM were swept with the same range as in the other GFM. These sweeps are displayed in fig. 4e and fig. 4f. Finally, the impact of the frequency droop gain, $K_{\text {droop}}$, is investigated. This parametric sweep is displayed in fig. $4 \mathrm{~g}$ and ranges from 0 to $10\left(100 \times 10^{6}\right)$.

\section{1) SM-GFM Network Parametric Sweep Results}

When analysing fig. $4 a$, it is seen that with an increasing length, the damping ratio of the electromechanical mode decreases from $16.84 \%$ to $12.88 \%$. In this test case the mode remains stable but in a different system, the impact of transmission line length might be more critical.

Altering the controller gains shows significant impact on the electromechanical mode in fig. $4 \mathrm{c}$ and fig. $4 \mathrm{~d}$. The gain $K_{I}$ is seen to cause small-signal instability of the electromechanical mode as it is increased whereas $K_{P}$ can fully damp the interaction, however the oscillation at $115 \mathrm{~Hz}$, seemingly related to network current dynamics, is brought towards the unstable region, as seen in fig. $4 \mathrm{~b}$.

\section{2) GFM-GFM Network Parametric Sweep Results}

The increase of the proportional and integral gains, $K_{P_{I}}$ and $K_{I_{1}}$, are seen to fully damp the electromechanical interaction and this time no adverse effect is found on any higher frequency modes. The droop gain sweep in fig. $4 \mathrm{~g}$ provides evidence of another highly impactful control parameter associated with the GFM, allowing for higher controllability of the electromechanical mode dynamics. Like the transmission line length sweep, it is seen that the electromechanical mode is brought towards the unstable region, potentially causing instability if this mode was initially closer to the y-axis.

\section{CONCLUSIONS}

This paper presents a preliminary investigation into interactions between SMs and GFMs with a focus on electromechanical modes. This is achieved with modular small-signal modelling, followed by eigenvalue analysis. The states corresponding to the electromechanical mode in the SM-GFM system were found to be those associated with the power loop of the GFM and the swing equation of the $\mathrm{SM}$, thereby confirming the presence of electromechanical interactions, similarly between two GFMs. Finally, a series of parametric sweeps are performed, offering an insight into the impact and flexibility that the GFM control provides for manipulating the electromechanical mode.

Small-signal instability is found to occur from high values of $K_{I}$ as the electromechanical mode traverses into the unstable region. In the case of $K_{P}$, this mode can be fully damped but doing so will bring a higher frequency oscillation towards instability. Additionally, for the GFM-
GFM network, it was found that the PI control gains of the droop-augmented GFM provided the potential to fully damp the electromechanical interaction with no significant effect on any higher frequency oscillations. However, increasing the frequency droop gain brought the electromechanical mode closer to the unstable region, similar to increasing the transmission line lengths.

\section{REFERENCES}

[1] International Panel on Climate Change (IPCC), "Special Report: Global Warming of 1.5 Degrees Celcius Summary for Policy Makers," IPCC, Geneva, 2018.

[2] F. Milano, F. Dörfler, G. Hug, D. J. Hill and G. Verbič, "Foundations and challenges of low-inertia systems (invited paper)," in 2018 Power Systems Computation Conference (PSCC), Dublin, 2018.

[3] M. Paolone, T. Gaunt, X. Guillaud, M. Liserre, S. Meliopoulos, A. Monti, T. Van Cutsem, V. Vijay and C. Vournas, "Fundamentals of power systems modelling in the presence of converter-interfaced generation," Electric Power Systems Research, vol. 189, p. 106811, 2020.

[4] X. Meng, K. Liu and Z. Liu, "A generalized droop control for gridsupporting inverter based on comparison between traditional droop control and virtual synchronous generator control," IEEE Transactions on Power Electronics, vol. 34, no. 6, pp. 5416-5438, 2019.

[5] M. Liu, S. Dutta, V. Purba, S. Dhople and B. Johnson, "A gridcompatible virtual oscillator controller: analysis and design," in 2019 IEEE Energy Conversion Congress and Exposition (ECCE), Baltimore, MD, 2019.

[6] H. P. Beck and R. Hesse, "Virtual synchronous machine," in 2007 9th International Conference on Electrical Power Quality and Utilisation, Barcelona, 2007.

[7] C. Collados-Rodriguez, M. Cheah-Mane, E. Prieto-Araujo and O. Gomis-Bellmunt, "Stability analysis of systems with high VSC penetration: where is the limit?," IEEE Transactions on Power Delivery, vol. 35, no. 4, pp. 2021-2031, 2019.

[8] R. Rosso, S. Engelken and M. Liserre, "Analysis of the behavior of synchronverters operating in parallel by means of component connection method (CCM)," in 2018 IEEE Energy Conversion Congress and Exposition (ECCE), Portland, OR., 2018.

[9] R. Rosso, S. Engelken and M. Liserre, "Robust stability analysis of synchronverters operating in parallel," IEEE Transactions on Power Electronics, vol. 34, no. 11, pp. 11309-11319, 2019.

[10] G. S. Pereira, V. Costan, A. Bruyére and X. Guillaud, "Simplified approach for frequency dynamics assessment of $100 \%$ power electronics-based systems," Electric Power Systems Research, vol. 188, p. 106551, 2020.

[11] U. Markovic, O. Stanojev, P. Aristidou, E. Vrettos, D. S. Callaway and G. Hug, "Understanding Small-Signal Stability of Low-Inertia Systems," IEEE Transactions on Power Systems.

[12] J.-S. Brouillon, M. Colombino, D. Gross and F. Dörfler, "The effect of transmission-line dynamics on globally synchronizing controller for power inverters," in 2018 7th European Control Conference (ECC), Limassol, 2018.

[13] P. Kundur, Power System Stability and Control, New York: McGraw-Hill, 1994.

[14] P. W. Sauer and M. A. Pai, Power System Dynamics and Stability, Urbana: Upper Saddle River, NJ, 1997.

[15] M. Reta-Hernandez, "Chapter 13 - Transmission Line Parameters," in Electric Power Generation, Transmission, and Distribution, Boca Raton, FL, CRC Press, 2012. 